EDITORIAL

\section{The national service framework for long term conditions: a novel approach for a "new style" NSF}

\section{Turner-Stokes}

$\mathrm{T}$ he setting of uniform standards presents a challenge for health and social care systems anywhere in the world. In the past 5 years, standards for the National Health Service (NHS) in England have been set out in a series of national service frameworks (NSFs). The latest in this series, the NSF for long term conditions, was released in March 2005 and presented some new challenges and novel solutions that have relevance for policy development in both the UK and other countries.

Previously published NSFs in the UK have provided standards for cancer, coronary heart disease, mental health diabetes, older people, children's services, and renal disease. Each set a series of 12 or more standards, and most included clearly defined national targets that service providers were obliged to meet. Many were easily measurable process targets, for example: "No patient should wait longer than 2 weeks for initial consultation in cases of suspected cancer", ${ }^{1}$ and "Thrombolysis should be given within 60 minutes of calling for professional help, in all eligible cases of acute myocardial infarction". ${ }^{2}$ Ring fenced funding was made available to help providers to achieve these targets, and a series of "milestones" was drawn up, defining the services that should be in place by specific dates.

While there can be no doubt that these targets have raised awareness and changed healthcare provision in many respects, their value has been challenged in many quarters. ${ }^{34}$ Importantly, by focusing on specific steps in the care pathway, they may divert attention from other critical steps. For example, a large study of over 5000 cases of breast cancer in the south of England showed that while the time to initial consultation had decreased, the time from first appointment to actual treatment had actually increased, and consequently total waiting times had changed little. ${ }^{5}$ In some more long term conditions, the focus on acute presentation may be even less productive. Reporting to the Public Administration
Select Committee in 2003, ${ }^{4}$ Richard Harrad, Clinical Director of the Bristol Eye Hospital, recorded that, to meet waiting time targets for new patients in diabetes clinics, 1000 follow up appointments were cancelled per month. Critical incident reporting over a 2 year period had identified 25 patients with diabetes or glaucoma who had lost vision as a result of consequent failure in follow up care.

These experiences have led the UK government to adopt a more "hands off" attitude, moving away from centrally allocated funding dependent on prescriptive targets, and allowing the NHS and social services more freedom in deciding how to meet national and local priorities across the whole care pathway. Future standards in the UK will have fewer national targets and greater emphasis on health outcomes as opposed to inputs. ${ }^{67}$ There will also be much greater user involvement in the setting of priorities and in service evaluation.

This latest NSF for long term conditions is therefore a "new style" NSF, set in that context. It goes forward together with a number of other initiatives that take a longer term view of health and social care centred on the needs and choices of the individual. ${ }^{8}$ In place of standards, targets and milestones, it has "quality requirements" to be implemented locally over a period of 10 years, and local bodies can set their own pace of change within this period, according to local priorities. No ring fenced funding is allocated; instead, implementation costs are expected to be met from uplifts in general health and social services funding, but will have to compete with other local priorities. The External Reference Group, appointed to provide advice to the Government on the NSF priorities, had very strong representation from service users and carers, and for the first time had a lay Chair - Diana Whitworth, then Chief Executive of Carers UK. Instead of focusing on specific areas of intervention, this NSF takes a more holistic approach, addressing all stages of the "care pathway" from initial presentation and diagnosis, through acute management and rehabilitation, into long term care and support, including palliative and end of life care. The ERG was tasked specifically with setting priorities for people with long term neurological conditions, and its membership was selected on this basis, but it was also asked to indicate where standards could be more generally applied to other long term conditions. Nevertheless, in implementation of this NSF, there is agreement on the importance of preserving the neurological focus and ensuring that it maintains its own discrete identity under the umbrella of the broader long term conditions programme ${ }^{10}$ in the UK.

Wherever they are applied, all health and social care standards should be based on the best available evidence, ${ }^{11}$ and a further challenge for this NSF has been the assimilation of research and evidence to underpin the recommendations. For a strongly user focused NSF, it was appropriate that the evidence presented should reflect the value placed on the opinions of users, carers, and professionals as well as formal research. Traditional research hierarchies such as those used by the National Institute for Clinical Excellence in the UK ${ }^{12}$ tend to emphasise research design, without due regard for the quality of the research or its applicability to the clinical question. ${ }^{13}$ Randomised, controlled trials have become the recognised gold standard for evidence relating to treatment efficacy, and are well suited to short term interventions in relatively homogeneous populations; however, they have well recognised limitations in more diverse populations with complex needs and cannot realistically be applied to all the questions that need to be answered.${ }^{14}$ In chronic conditions, where the important outcomes are quality of experience over a life long time scale, designs such as longitudinal observational studies or qualitative research techniques are more likely to be appropriate for many of the questions. However, they must be conducted rigorously, with all possible steps taken to reduce bias.

A new typology for research and evidence was therefore developed by the Research and Evidence Group for this NSF, which recognises the breadth of research design required in this area of health and social care. Importantly, it takes account of the opinions and experience of service users, and their families and carers, as well as the views of professionals. It is based on the principle that qualitative, quantitative and mixed studies can have equal validity when used in the appropriate context, rather than suggesting that there is an implicit hierarchy among 


\begin{tabular}{ll} 
Table 1 Summary of evidence typology for the NSF for long term conditions \\
\hline $\begin{array}{l}\text { Expert evidence } \\
\text { Opinion/experience }\end{array}$ & Of users, carers (E1) or professionals (E2) \\
$\begin{array}{l}\text { Research based evidence } \\
\text { Design }\end{array}$ & \\
Primary research & Quantitative (P1), qualitative (P2) or mixed methods (P3) \\
Secondary research & Meta-analysis (S1) or other secondary analysis (S2) \\
Reviews & Systematic (R1), or other descriptive reviews (R2) \\
Quality assessment & Rated on five parameters (scored out of 10) \\
Applicability & Direct (evidence from within long term neurological conditions) or \\
& indirect (extrapolated evidence from other conditions)
\end{tabular}

research designs, ${ }^{15}$ and it puts the emphasis on the quality of the study design, and the integrity of its conclusions and their relevance to the population served by this NSF.

To assign the typology: (a) each piece of evidence referenced in the NSF has been given an " $\mathrm{E}$ " (expert) or an " $\mathrm{R}$ " (research) rating; (b) each piece of research based evidence in the NSF was awarded a rating based on three domains (design, quality, and applicability) (see table 1); and (c) each quality requirement was then presented with a grade of recommendation reflecting the extent, quality, and applicability of expert and research evidence.

The scheme for quality assessment is a simplified rating, based broadly on the methods developed by Van Tulder and colleagues for systematic reviews within the Cochrane Library ${ }^{16}$ and adapted by Steultjens et al for other designs. ${ }^{17}$ Any such quality rating inevitably has an element of subjectivity, and to reduce variation it is therefore recommended that typology should be assigned by at least two raters. The Research and Evidence Subgroup of the ERG evaluated over 600 articles and pieces of evidence to assimilate the evidence base for the 11 quality requirements. The process by which the typology was developed, validated, and applied is currently in preparation for publication.
It is intended that this approach will mark a significant departure from traditional evidence evaluation and that it will take the research community a further step towards recognising the broader church of research methodologies needed to reflect the real life experience of individuals and their families who need to use our health and social services on a life long basis.

\section{ACKNOWLEDGEMENTS}

I would like to thank the other members of the Research and Evidence Subgroup of the NSF ERG who were involved in development and evaluation of the evidence typology. Financial support for typology development was provided through a grant from the Department of Health Research and Development programme. Support for the preparation of this manuscript was kindly provided by the Luff Foundation.

\section{J Neurol Neurosurg Psychiatry 2005;76:901-902.}

doi: 10.1136/jnnp.2005.066472

Correspondence to: Professor Lynne TurnerStokes, Director, Regional Rehabilitation Unit, Northwick Park Hospital, Watford Road,

Harrow, Middlesex HA1 3UJ, UK;

lynne.turner-stokes@dial.pipex.com

Received 28 February 2005

In revised form 28 February 2005

Accepted 2 March 2005

Competing interests: As Deputy Chair of the NSF the author naturally has a desire to see that the recommendations are successfully delivered. Any opinions expressed in this article are the author's personal viewpoints and are not necessarily shared by the ERG membership or the Department of Health NSF team.

\section{REFERENCES}

1 Department of Health. The NHS cancer plan. London: DoH, 2000.

2 Department of Health. National service framework for coronary heart disease. London: DoH, 2000.

3 Yoong KK, Heymann T. Target centred medicine: targets can seriously damage your health. BMJ 2003;327:20.

4 Public Administration Select Committee. On target? Government by measurement. London: The Stationary Office, 2003

5 Robinson D, Bell CM, Moller H, et al. Effect of the UK government's 2-week target on waiting times in women with breast cancer in southeast England. B J Cancer 2003;89:492-6.

6 Department of Health. National standards, local action: health and social care standards and planning framework. London: DoH, 2004.

7 Department of Health. Standards for better health. London: DoH, 2004

8 Department of Health. Building on the best: choice, responsiveness and equity in the NHS London: DoH, 2003.

9 Department of Health. The new vision for adult social care: results of the SCIE survey and the development of a Green Paper, 2005. www.dh.gov.uk/PolicyAndGuidance/ HealthAndSocialCareTopics/SocialCare/ $/ f_{s} / e n$.

10 Department of Health. Supporting people with long term conditions: An NHS and social care model for improving care for people with long term conditions. London: DoH, 2005.

11 Sackett DL, Rosenberg WMC, Gray JAM, et al. Evidence based medicine: what it is and what it isn't. BMJ 1996;312:71-2.

12 National Institute for Clinical Excellence. Guide to the methods of technology appraisal. London: NICE, 2004.

13 Royal College of Physicians. National clinical guidelines for stroke. London: RCP, 2002.

14 Whyte J. Traumatic brain injury rehabilitation: are there alternatives to randomised controlled clinical trials? Arch Phys Med Rehab 2002;83:1320-2.

15 Malterud K. The art and science of clinical knowledge: evidence beyond measures and numbers. Lancet 2001;358:397-400.

16 van Tulder MW, Assendelft WJ, Koes BW, et al. Method guidelines for systematic reviews in the Cochrane Back Review group for Spinal Disorders. Spine 1997;22:2323-30.

17 Steultiens EMJ, Dekker J, Bouter LM, et al. Occupational therapy for rheumatoid arthritis. The Cochrane database of systematic reviews. Oxford: Update Software, 2003. 
Parkinson's disease

\section{Cholinesterase inhibitors for treatment of dementia associated with Parkinson's disease}

\section{J L Cummings}

\section{Improving cognitive function and reducing neuropsychiatric symptoms}

$\mathrm{P}$ atients with Parkinson's disease (PD) have a significant risk of developing dementia in the course of their illness. Cross sectional studies suggest a dementia prevalence rate of $30 \%$ to $40 \% .^{1}$ Longitudinal studies indicate that the cumulative frequency of dementia in patients with $\mathrm{PD}$ is $60 \%$ to $80 \%{ }^{1}$ The risk of dementia for individuals with PD is approximately six fold greater than that of age matched controls. The increased rate of dementia in PD reflects the substantial elevation of risk for dementia in patients with $\mathrm{PD}$ above the risk posed by Alzheimer's disease in this same population. Recent studies demonstrate a strong correlation between the occurrence of Lewy body pathology and severity of dementia in patients with $\mathrm{PD} .^{2}$ The sensitivity, specificity, positive predictive value, and negative predictive value of cortical Lewy bodies for dementia exceeds that of senile plaques or neurofibrillary tangles, ${ }^{2}$ again suggesting that the dementia of $\mathrm{PD}$ is related to the unique pathology of PD and in most cases does not reflect co-occurring Alzheimer's disease. There is a marked cholinergic deficit in patients with PD and dementia indicating that there is a cholinergic contribution to the cognitive decline. ${ }^{3}$

The presence of a cortical cholinergic deficit in patients with PD and dementia suggests that treatment with a cholinesterase inhibitor (ChE-I) may be beneficial. In this issue, Ravina and colleagues ( $p p$ 934-9 $)^{4}$ report the results of a small double blind placebo controlled crossover study of donepezil for treatment of dementia in PD. Twentytwo patients participated in the study, which consisted of two treatment periods of 10 weeks separated by a 6 week wash out interval. The primary outcome measure was the Alzheimer's Disease Assessment Scale Cognitive Subscale (ADAScog). While receiving donepezil, patients evidenced a mean 1.9 point improvement on the ADAScog, a change that did not reach statistical significance. The secondary outcome measures included the Mini Mental State Examination (MMSE), Mattis Dementia Rating Scale (MDRS), Clinical Global Impression of Change (CGI), and Brief Psychiatric Rating Scale (BPRS). There was a significant 2 point benefit on the MMSE and a significant 0.37 point improvement on the CGI. No beneficial or detrimental changes were observed on either the MDRS or the BPRS. All but three patients completed the trial (two dropouts during the donepezil treatment period and one dropout during placebo treatment), and no statistically significant differences in adverse events were found. Donepezil did not worsen parkinsonism. The authors concluded that donepezil may produce a modest cognitive benefit in patients with PD and dementia. Although the primary outcome measure did not distinguish the two groups there was a trend for improvement following treatment with donepezil and two secondary measures including the MMSE showed a statistically significant benefit. The power to detect differences of small magnitude is compromised by the limited sample size.

This study with donepezil must be seen in the context of the much larger double blind placebo controlled parallel group study of rivastigmine for the treatment of PD with dementia recently reported by Emre and colleagues. ${ }^{5}$ This trial enrolled 541 patients of whom 410 completed the study. Statistically significant benefit was seen for both primary measures (ADAScog and the CGI) as well as the secondary measures including the Alzheimer's Disease Cooperative Study Activities of Daily Living Scale, the Neuropsychiatric Inventory, MMSE, Cognitive Drug Research power of attention test, verbal fluency test, and clock drawing test. No worsening of parkinsonism was noted on formal rating scales but adverse event reporting suggested an increase in tremor in the patients treated with rivastigmine. Tremor was rarely sufficient to lead to treatment discontinuation. The more favourable outcome of this study compared to that reported by Ravina et $\mathrm{al}^{4}$ may be ascribed to the larger size and greater power of the trial, the use of rivastigmine rather than donepezil for acetylcholinesterase inhibition, or other differences in trial design and analysis. The study by Emre and colleagues ${ }^{5}$ adds substantial weight to the suggestion that cholinesterase inhibitors may be beneficial in the treatment of dementia associated with PD.

A major caveat in clinical trials for the treatment of dementia associated with PD concerns the measurement of cognitive deficits. In this patient group executive deficits predominate and are more marked than the classical instrumental deficits involving memory, language, and praxis characteristic of Alzheimer's disease. The MMSE and ADAScog are relatively sensitive to the cognitive changes of Alzheimer's disease but both instruments lack executive measures. Use of these instruments to render comparison with treatment responses in Alzheimer's disease feasible may compromise detection of the impact of cholinesterase inhibitors on executive functions mediated by frontal subcortical circuits. Ravina and colleagues $^{4}$ included the MDRS-an instrument with measures of executive function-and noted no effect from treatment with donepezil. Emre et al included verbal fluency and clock drawing tasks that require executive function and observed improvement in both measures following treatment with rivastigmine. Inclusion of measures of executive function is critical to a comprehensive assessment of the effects of cholinesterase inhibitors and other agents potentially useful in the treatment of dementia associated with PD.

The two recent studies discussed above add to a growing literature suggesting that cholinesterase inhibitors improve cognitive function in patients who have dementia associated with PD. ${ }^{6-8}$ Several studies also indicate that cholinesterase inhibitors may reduce neuropsychiatric symptoms in PD, particularly hallucinations and delusions. ${ }^{78}$ Dementia is a significant risk factor for neuropsychiatric symptoms in PD.

The growing literature on the potential utility of cholinesterase inhibitors in dementia associated with PD suggest that these agents may be helpful in improving cognitive function and reducing neuropsychiatric symptoms. Additional research is needed to determine whether all cholinesterase inhibitors are of equal efficacy in this setting, the duration of benefit to be expected, and the interaction with dopaminergic 
agents and psychotropic drugs commonly used in this patient population.

\section{ACKNOWLEDGEMENTS}

Dr Cummings is supported by the National Institute on Aging (P50 AG 16570), the Alzheimer's Disease Research Centers of California, and the Sidell-Kagan Foundation.

\section{$J$ Neurol Neurosurg Psychiatry 2005;76:903-904. \\ doi: 10.1136/jnnp.2004.061499}

Correspondence to: Dr Jeffrey L Cummings, Reed Neurological Research Center, 710 Westwood Plaza, David Geffen School of Medicine at UCLA, Los Angeles, CA 900951769, USA; jcummings@mednet.ucla.edu
Competing interests: Dr Cummings has served as a consultant or performed research for AstraZeneca, Aventis, Bristol Myers-Squibb, Eisai, Janssen, Lilly, Lundbeck, Memory, Merz, Neurochem, Novartis, Ono, Pfizer, Praecis, and Wyeth.

\section{REFERENCES}

1 Aarsland D, Andersen K, Larsen JP, et al. Prevalence and characteristics of dementia in Parkinson disease: an 8-year prospective study. Arch Neurol 2003:60:387-92.

2 Hurtig H, Trojanowski JQ, Galvin J, et al. Alphasynuclein cortical Lewy bodies correlate with dementia in Parkinson's disease. Neurology 2000:54:1916-21.

3 Bohnen NI, Kaufer DI, Ivanco LS, et al. Cortical cholinergic function is more severely affected in parkinsonian dementia than in Alzheimer disease: an in vivo positron emission tomographic study. Arch Neurol 2003;60:1745-8.

4 Ravina B, Putt M, Siderowf A, et al. Donepezil for dementia in Parkinson's disease: a randomised, double blind, placebo controlled, crossover study. J Neurol Neurosurg Psychiatry 2005;76:934-9.

5 Emre $M$, Aarsland D, Albanese A, et al. Rivastigmine for dementia associated with Parkinson's disease. N Engl J Med 2004;351:2509-18.

6 Aarsland D, Laake K, Larsen JP, et al. Donepezil for cognitive impairment in Parkinson's disease: a randomised controlled study. J Neurol Neurosurg Psychiatry 2002;72:708-12.

7 Aarsland D, Hutchinson M, Larsen JP. Cognitive, psychiatric and motor response to galantamine in Parkinson's disease with dementia. Int J Geriatr Psychiatry 2003;18:937-41

8 Hutchinson M, Fazzini E. Cholinesterase inhibition in Parkinson's disease. J Neurol Neurosurg Psychiatry 1996;61:324-5.

\section{Surgery for movement disorders: new applications?}

\section{P Limousin-Dowsey, S Tisch}

\section{The effect of basal ganglia surgery on Tourette syndrome, tardive} dyskinesias, and myoclonus-dystonia

$\mathrm{T}$ he last 15 years have been a period of significant advances in functional neurosurgery for movement disorders largely due to the development of the technique of deep brain stimulation (DBS) and the refinement of the targeting procedure. Deep brain stimulation is now well established for the treatment of Parkinson's disease, tremor, and primary dystonia, and is progressively being used for new applications. In this issue, Houeto et al ${ }^{1}$ ( $p p$ 992-5) present a case report on the effect of basal ganglia surgery on Tourette syndrome, Lenders et $a l^{2}(p$ 1039) on tardive dyskinesias, and Magarinos-Ascone et $a l^{3}$ ( $p p$ 98991) on myoclonus-dystonia.

Tourette syndrome is a disabling movement disorder-the signs include motor and vocal tics and various behavioural dysfunction. Ablative procedures have been carried out in the frontal area or the thalamus. An earlier report has shown an improvement of tics in three patients following thalamic DBS. ${ }^{4}$ In the present study Houeto et al ${ }^{1}$ compared the effect in two targets-the centromedian-parafascicular complex of the thalamus (Ce-Pf) and the antero-medial part of the internal globus pallidus (GPi) - both part of the limbic basal ganglia-thalamo-cortical loop. ${ }^{1} \quad$ They conducted a double blind study of four periods with different conditions of stimulation: off-stimulation, GPi stimulation, Ce-Pf stimulation, and GPi and Ce-Pf stimulation. GPi stimulation or Ce-Pf stimulation improved tic severity by $70 \%$, improved coprolalia, and eliminated self injuries. Emotions and depression were improved with Ce-Pf stimulation. Lenders et $a l^{2}$ report on a patient with tardive dyskinesias, predominantly dyskinetic in the right hemibody treated by left pallidotomy with 5 years follow up of good improvement. ${ }^{2}$ Few cases had been previously reported. Magariños-Ascone et $a^{3}$ report the improvement of a myoclonus-dystonia syndrome by GPi stimulation, confirming earlier reports.

These studies are all case reports and although encouraging the results are limited by virtue of the sample size. In rare disorders such as these a multicentre study would allow a larger number of patients to be followed. The best target for Tourette syndrome certainly needs additional data to support this early finding. Other issues exist with Tourette syndrome and tardive dyskinesias that often include debilitating psychiatric problems. Patients with psychiatric problems may become preoccupied with the DBS implanted system and could even develop delusions that DBS is exerting "mind control". Therefore it is particularly important in those patients to have a multidisciplinary team to discuss the specific ethical issues, establish strict criteria of selection, and evaluate the impact of surgery on all aspects of the disease. For some conditions improved rating scales may need to be developed to better quantify clinical outcome after surgery. The impact on cognitive and behaviour functions also need to be carefully documented. Unilateral pallidotomy involves a one off procedure and no implanted equipment, and might still have a place in experienced hands and selected cases like Lenders et al. ${ }^{2}$ Many questions remain on the mechanism of action of basal ganglia and thalamic surgery, and in particular how can they improve such a variety of conditions.

J Neurol Neurosurg Psychiatry 2005;76:904. doi: 10.1136/jnnp.2004.062117

a................

Authors' affiliations

P Limousin-Dowsey, S Tisch, Institute of Neurology, London, UK

Correspondence to: P Limousin-Dowsey, Sobell Department of Motor Neuroscience and Movement Disorders, Institute of Neurology, Box 146, Queen Square, London WCIN 3BG, UK; p.limousin@ion.ucl.ac.uk

\section{REFERENCES}

1 Houeto JL, Karachi CC, Mallet L, et al. Tourette's disorder and deep brain stimulation. J Neurol Neurosurg Psychiatry 2005;76:992-5.

2 Lenders $M$, Vergouwen $M$, Jansen Steur $E$, et al. Long term results of unilateral posteroventral pallidotomy for antipsychotic drug-induced tardive dyskinesia. J Neurol Neurosurg Psychiatry 2005;76:1039.

3 Magariños-Ascone CM, Regidor I, MartínezCastrillo JC, et al. Pallidal stimulation relieves myoclonus-dystonia syndrome. J Neurol Neurosurg Psychiatry 2005;76:989-91

4 Visser-Vandewalle V, Temel Y, Boon P, et al. Chronic bilateral thalamic stimulation: a new therapeutic approach in intractable Tourette syndrome. Report of three cases. J Neurosurg 2003;99:1094-100. 
Stroke

\section{Ultrafast magnetic resonance imaging protocols in stroke}

\section{E B Ringelstein}

Profound changes in the protocols for the management of acute stroke patients irrespective of whether ischaemic or haemorrhagic in origin

A lthough the approval of recombinant tissue plasminogen activator (rtPA) for the treatment of acute ischaemic stroke has been based on the primary imaging technique of conventional cerebral computerised tomography (CT), ${ }^{1}$ newer and more refined magnetic resonance (MR) techniques are unrelentlessly extending into this particular field of emergency medicine, stimulated by the important hope that these new techniques could help to prolong the therapeutic time window beyond the 3 hour limit in selected patients. Several recent papers have clearly demonstrated that the mismatch concept, ${ }^{2}$ though leading to diagnostic pitfalls in a small number of cases as well, seems to be helpful in selecting patients for "late" fibrinolysis. It has been shown that the rapidity with which the inevitable core infarction augments into the tissue at risk (= penumbra) does not only depend on external parameters like perfusion pressure, blood glucose level, body temperature, or oxygen saturation (to mention only a few), but is strongly influenced by individual, predefined anatomical features of the cerebral vasculature like the configuration of the circle of Willis and the collateralising potential of the leptomeningeal anastomoses at the cortical level. ${ }^{3}$

The indication for systemic fibrinolysis in acute stroke is not dependent on the angiological proof of an intracranial arterial occlusion. The conflict between the desire to base fibrinolysis on pathophysiologically relevant knowledge, and the urge of the therapeutic time window with an exponentially decreasing benefit from lysis over time led to a decision in favour of the time requirements. It could be argued, however, that patients without a visible arterial occlusion would not need fibrinolysis at all, and should not be exposed to its intrinsic risks.

With this in mind the idea to subject nearly every acute stroke patient to an ultrafast MR imaging protocol including $\mathrm{T} 1, \mathrm{~T} 2$, fluid attenuated inversion recovery (FLAIR), and diffusion weighted imaging (DWI) sequences in addition to three dimensional time-of-flight MR to visualise the major cerebral arteries appears very attractive even though this technology and the corresponding expertise is not available yet over 24 hours in most places. Until now, the most convincing argument against this refined diagnostic work-up of the acute stroke patient was the limitation of time. This is no longer valid, however, and postprocessing efforts lasted only 7 minutes, during which time the patient was returned to the ward. With this ultra fast MRI technique, even the handling of restless and uncooperative patients did not seem to be a problem anymore-another important argument often posed against MR imaging in the very acute phase of stroke.
A technique like the one described by U-King-Im et al (this issue, pp 1002-5), supplemented by an additional haemosiderin-sensitive gradient echo sequence for the detection of previous haemorrhages might initiate profound changes in the protocols for the management of acute stroke patients irrespective whether ischaemic or haemorrhagic in origin. Realistic hopes are that: (1) ideal candidates for fibrinolysis could be pinpointed; (2) the time window for fibrinolysis could be prolonged on an individual basis; (3) the risk of intracerebral haemorrhage could be reduced by excluding patients without a visible arterial lesion; and (4) patients with multiple haemosiderin remnants indicating previous brain haemorrhages could be excluded as well, because of their excessive bleeding risk. In patients with peracute brain haemorrhage, recombinant activated Factor VII could be applied to stop the growth of the haematoma. ${ }^{4}$ If this works, only two major obstacles still seem to be present: the patients' cardiac pacemakers and defibrillators, and the costs.

J Neurol Neurosurg Psychiatry 2005;76:905. doi: 10.1136/jnnp.2004.060871

Correspondence to: Professor E B Ringelstein, Klinik und Poliklinik für Neurologie,

Universitätsklinikum Münster, Albert-

Schweitzer-Str 33, 48149 Münster, Germany

D-48129; ringels@uni-muenster.de

\section{REFERENCES}

1 NINDS (National Institute of Neurological Disorders and Stroke rt-PA) Stroke Study-Group. Tissue Plasminogen Activator for Acute Ischaemic Stroke. In: N Engl J Med 1995;333:1581-7.

2 Röther J, Schellinger PD, Gass A, for the Kompetenznetzwerk Schlaganfall Study Group, et al. Effect of Intravenous Thrombloysis on MRI Parameters and Functional Outcome in Acute Stroke $<6$ Hours. Stroke 2002;33:2438-45.

3 Ringelstein EB, Biniek R, Weiller C, et al. Type and extent of hemispheric brain infarctions and clinical outcome in early and delayed middle cerebral artery recanalization. Neurology 1992;42:289-98.

4 Mayer SA, Brun NC, Broderick J, et al. Europe/ AustralAsia NovoSeven ICH Trial Investigators. Safety and feasibility of recombinant factor Vlla for acute intracerebral hemorrhage. Stroke 2005;36:74-9. 